\title{
Cien años de Metafísica dentro de la tradición filosófica analítica. Introducción a la sección monográfica sobre metafísica analítica contemporánea
}

\author{
A Hundred Years of Metaphysics within the Analytic Tradition. \\ Introduction to the Monographic Section on \\ Contemporary Analityc Metaphysics
}

\author{
Carlo Rossi \\ Departamento de Filosofía, Universidad Adolfo Ibáñez, Chile \\ carlo.rossi.f@uai.cl
}

\section{Introducción}

El inicio de la tradición filosófica analítica estuvo marcado, entre otros hechos, por el rechazo de autores como Gottlob Frege, Bertrand Russell, G.E.E. Moore y Ludwig Wittgenstein al Psicologismo e Idealismo que dominaba una buena parte de la escena filosófica a fines del s. XIX y a comienzos del s. XX en Inglaterra y Alemania. Sin embargo, posteriormente y por influjo del Empirismo Lógico, la reacción a estas posiciones dominantes se tradujo en una cierta hostilidad hacia la Metafísica como disciplina filosófica. La Filosofía, dirán autores como Carnap (1928) y Ayer (1936), tendría por objeto depurar y sistematizar el aparataje conceptual empleado por la ciencia natural en su comprensión del mundo. Pero dadas las altas exigencias del criterio para evaluar el significado de un enunciado propuesto por el Empirismo Lógico, ninguna parte de la Filosofía cuyos enunciados no pudiesen ser verificados empíricamente se juzgaría como dotada de significado, en el sentido técnico del término.

Como tal vez cabía esperar, tal hostilidad con el paso del tiempo fue decayendo en el seno de la tradición analítica, a la vez que se incrementaba su apertura a problemas que podrían considerarse como propios de la Metafísica y la Ontología. Dos hechos salientes se pueden notar en este sentido. El primer de ellos es la publicación por parte de Quine, en 1953, del paper “On What There Is?”(1953). Si bien Quine no se podría considerar

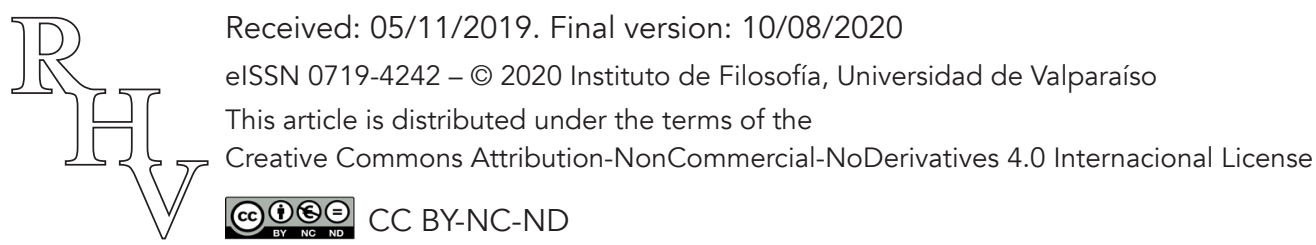


precisamente como un filósofo cercano a una concepción robusta y autónoma de la Metafísica, su teoría sobre compromiso ontológico en tal articulo volvió a dotar a las preguntas de carácter ontológico y metafísico de la respetabilidad que habían perdido en décadas pasadas en ciertos círculos filosóficos. El segundo de ellos - y a pesar de las objeciones críticas provistas por Quine mismo (1953a) - es el desarrollo de la Lógica Modal a partir de las contribuciones seminales de Barcan (1946) y Kripke (1959; 1963), el cual a su vez luego posibilitó en décadas siguientes el florecimiento de la Metafísica Modal. Prueba de ello son las ideas desarrolladas por el mismo Kripke (1971; 1980), Lewis (1973; 1986), Plantinga (1974), Adams (1974; 1979) y Armstrong (1989), sólo por nombrar a algunos participantes más connotados de este debate.

El presente número especial tiene la finalidad de presentar contribuciones originales a algunos de los principales debates metafísicos contemporáneos dentro de la tradición filosófica analítica, cubriendo temas tan variados como la naturaleza de las propiedades universales y las propiedades intrínsecas, la relación de fundamentación e identidad, paradojas de composición material y locación, la relatividad conceptual y nuestros esquemas ontológicos, entre otros. A grandes rasgos, las cinco contribuciones de este número se agrupan en dos grandes familias de temas: la Metafísica de los Objetos Materiales y la Metafísica de Propiedades. La década pasada tuvo lugar un auténtico renacimiento del interés por distintas cuestiones sobre la Metafísica de Objetos Materiales. Si tal vez ciertas contribuciones seminales a este debate, como las de Chisholm (1976), Lewis (1986), van Inwagen $(1981,1990)$ o Sider (2001), adoptaron un tono decididamente controversial y marcadamente comprometido con las teorías defendidas, contribuciones más recientes se han abocado a proveer un entendimiento más profundo y detallado tanto de las diferentes teorías disponibles (con sus correspondientes implicancias), como de la propia índole filosófica de estos debates. Este afán se ha servido, por una parte, de una amplia utilización de los recursos provistos por la Mereología y Teorías de Locación, y por otra, por la floreciente literatura sobre la metametafisica de estos debates (Chalmers, Manley y Wassermann 2009; Correia y Schnieder 2012; Kleinschmidt 2014; Cotnoir y Baxter 2014; Korman 2015; Gilmore 2018; Brewer y Cumpa 2019; Varzi 2020).

\section{Metafísica de Objetos Materiales}

En "Composición, Identidad y Cardinalidad", la primera contribución de este número, Sebastián Briceño argumenta contra la relativamente popular tesis de la Composición como Identidad (Baxter 1988; Baxter 2014; Wallace 2011; Wallace 2014; Bohn 2014; Bricker 2016). De acuerdo con esta tesis, un todo no sería nada por sobre la suma de sus partes, de manera que la relación de composición sería identidad en algún sentido por especificar. Briceño toma partido en este debate por una posición que trata las relaciones de identidad y composición como lógica y ontológicamente distintas. Tal posición cuando menos puede ser rastreada hasta Parménides y más recientemente a Leibniz y Russell. La 
idea a defender sería que la identidad es una relación primitiva reflexiva, simétrica y transitiva que un objeto solo tiene consigo mismo y con ningún otro objeto. La composición, en cambio, es una relación instanciada, por una parte, por una pluralidad de objetos, y por otra, por un solo objeto, del cual los primeros se dicen partes del segundo. Entre otras cosas, esto implica que para que un objeto se considere parte de una fusión o todo, debe existir también un tercer objeto distinto a éste que también sea parte de dicho todo. En otras palabras - y tal como dictaminan los distintos axiomas de Suplementación-debe existir una pluralidad de objetos. Tras examinar el estado de la cuestión e identificar y evaluar los principales argumentos en juego, Briceño concluye que la Composición como Identidad, tanto en su versión fuerte como en su versión débil, no puede dar cuenta satisfactoriamente de las intuiciones más plausibles que se comparten en el debate acerca de la identidad y la composición.

Las otras dos contribuciones que se incluyen en el área de la Metafísica de Objetos Materiales tratan acerca del Problema de la Fundamentación (Grounding Problem). La pregunta central que hace este problema es cómo exactamente hemos de concebir la relación de fundamentación entre las propiedades de tipo clasificatorio o "sortal" (sortals) de un objeto material respecto de sus propiedades de tipo no clasificatorio. El Problema de la Fundamentación se vuelve aún más acucioso cuando consideramos las dificultades que trae consigo la posibilidad de la co-locación, esto es, la posibilidad de que dos objetos materiales que ejemplifican distintos tipos o propiedades clasificatorias ocupen la misma región espacial al mismo tiempo. Esto debido a que resulta prima facie difícil de apreciar cómo, si las propiedades clasificatorias se fundan en las propiedades intrínsecas de un objeto material, la misma base de propiedades intrínsecas que encontramos en una región espacial podría fundar dos objetos materiales que ejemplifican distintas propiedades clasificatorias.

Ahora, antes de introducir las contribuciones de Ezequiel Zerbudis y Marta Campdelacreu a este debate, es necesario hacer una aclaración adicional acerca de la relación de fundamentación como un tipo de relación que nos proporciona explicaciones metafísicas no causales sobre el mundo. Ejemplos que ilustrarían el trabajo que esta noción supuestamente hace son enunciados tales como "El conjunto cuyo único miembro es Sócrates existe en virtud de la existencia de Sócrates", "Un acto es querido por los dioses por ser piadoso", y "La mesa existe debido a la existencia de las partes que la componen". Las relaciones de fundamentación se consideran necesarias y constituyen un orden parcial estricto en virtud del hecho de ser irreflexivas, asimétricas y transitivas. Dicho esto, puede ser útil distinguir además entre la noción de fundamentación como una relación explicativa o bien como una relación no explicativa que respalda las explicaciones de fundamentación que podemos invocar (Bliss y Trogdon 2014). Los filósofos que han defendido la primera opción tratan la noción de fundamentación como un conectivo para oraciones no extensional (Correia 2010; Fine 2012). Los defensores de esta opción permanecen neutrales sobre la existencia de entidades, como hechos o proposiciones, que estarían 
conectadas cuando invocamos la relación de fundamentación en nuestras explicaciones acerca del mundo. Por otra parte, los defensores de la segunda opción regimientan el uso que hacemos del predicado de fundamentación en nuestras explicaciones por medio de un predicado relacional que designa dicha relación, cuyos relata podrían ser hechos, proposiciones y cosas según sea la teoría (Schaffer 2009; Rosen 2010; Audi 2012).

En su artículo "Una Defensa de la Solución Conceptualista al Problema de la Fundamentación para Objetos Coincidentes", Zerbudis defiende la alternativa ofrecida por el Conceptualismo para resolver el Problema de Fundamentación. La intuición que explota la solución conceptualista aquí es que las propiedades clasificatorias de un objeto material dependen en última instancia de nuestras prácticas clasificatorias como sujetos de experiencia. En este sentido - y más precisamente - el hecho que ciertos objetos sean considerados como ejemplificando una cierta propiedad clasificatoria dependerá del hecho de que son concebidos como tales por un sujeto, quedando afectos a las reglas que rigen las condiciones de aplicación (o condiciones iniciales) del concepto clasificatorio en cuestión. En particular, Zerbudis intenta dar una respuesta a dos de las objeciones desarrolladas por Bennett (2004) contra la posición conceptualista. Estas objeciones urgen al conceptualista a clarificar distintos problemas que surgen a partir de la introducción de las condiciones iniciales. La primera de ellas, Validez Conceptual, ataca la supuesta tesis defendida por la conceptualista de acuerdo con la cual es analítica o conceptualmente verdadero que se siga la existencia de dos o más objetos materiales de tipo diverso a partir de la satisfacción de las condiciones iniciales estipuladas para un concepto clasificatorio aplicable a objetos materiales. La segunda, Instanciación, sostiene que la conceptualista no puede dar una respuesta satisfactoria a la pregunta acerca de qué exactamente-y cómo, si es que efectivamente existe-instancia las propiedades no clasificatorias incluidas en las condiciones iniciales de un determinado concepto clasificatorio. Zerbudis argumentará en su artículo que ambas objeciones finalmente no socavan la posición conceptualista, puesto que o bien atribuyen al Conceptualismo supuestos que no tendría por qué adoptar, o bien porque le restringen ciertos recursos legítimos a los cuales también recurren otras posiciones rivales del presente debate.

Por su parte, Campdelacreu en "Una nueva solución al problema de la fundamentación" se propone desarrollar una respuesta pluralista y de alcance más general al Problema de la Fundamentación para objetos materiales co-localizados que la elaborada por Sutton (2012). Con solución pluralista me refiero aquí a un tipo de solución que admite la existencia de dos o más objetos para una misma región espacio-temporal para la versión del Problema de la Fundamentación que supone co-localización de objetos materiales. De acuerdo con Campdelacreu, si bien la respuesta favorecida por Sutton es esencialmente correcta, dicha respuesta no podría aplicarse a cierto tipo de casos presentados por Campdelacreu (2016) y por tanto adolecería de la generalidad deseable que una respuesta al Problema de la Fundamentación debiera tener. El presente artículo distingue tres casos distintos de co-localización, la cual se asume para toda la existencia temporal del par de 
objetos en cuestión, a partir de dos variantes de composición material, a saber, composición extrínseca y composición intrínseca. Un objeto estaría extrínsecamente compuesto si y solo si su composición y existencia está fundamentada al menos parcialmente en relaciones que sus partes mantienen con entidades que no son parte de dicho objeto. En cambio, en el caso de la composición intrínseca, la composición y existencia del objeto en cuestión se funda únicamente en las partes que lo componen, con prescindencia de las relaciones que estas partes puedan tener con otras entidades. De este modo, para todo par de objetos materiales co-localizados, o bien los dos objetos están compuestos extrínsecamente, o bien sólo uno de ellos, o bien los dos están compuestos intrínsecamente. La virtud de la solución de Campdelacreu estriba en que no sólo ofrece una respuesta para los dos primeros casos, sino también para el tercero, el cual se podría considerar como el más problemático para quienes soluciones pluralistas al Problema e la Fundamentación. Además, esta respuesta ofrecería ventajas ostensibles por sobre respuesta rivales como las de Fine (2003; 2008) y Saenz (2015), que también al mismo nivel de generalidad.

\section{Metafísica de Propiedades}

Los dos artículos que se incluyen en este volumen sobre Metafísica de Propiedades presentados por Gonzalo Rodriguez-Pereyra y Robert Garcia tratan respectivamente acerca de la posibilidad de propiedades universales indiscernibles y sobre el fenómeno del carácter denso de las propiedades. Si bien en los orígenes de la tradición filosófica analítica es posible encontrar contribuciones relevantes acerca del status ontológico de las propiedades universales, tales como Frege (1891; 1892), Russell (1912; 1912a) y Ramsey (1925), los temas tratados por las contribuciones incluidas en este volumen se conectan más directamente con Black (1952), Williams (1953) y posteriormente con Armstrong (1978). Ambos artículos, entre otros temas, intentan responder a preguntas sobre la naturaleza de las propiedades inmanentes y de las propiedades particulares no-compartibles (tropos), sobre la distinción ente particulares y universales y la viabilidad de la Teoría de Cúmulos o Bundle Theory en su versión de universales inmanentes (o realista, como la denomina García) y en su versión de tropos. ${ }^{1}$ En el artículo de Rodríguez-Pereyra "Universales Indiscernibles" encontramos un caso persuasivo a favor de la existencia de universales inmanentes perfectamente similares. Rodríguez-Pereyra sostiene que a pesar de que tradicionalmente se ha considerado que no puede haber universales perfectamente similares dada la ley de la Identidad de los Indiscernibles (puesto que ésta excluye la posibilidad de que existan dos entidades numéricamente distintas, pero perfectamente similares), pareciera en principio que tal consideración no es válida para el caso de los universales inmanentes. Rodríguez-Pereyra sustenta su caso a favor de los universales inmanentes perfectamente similares con dos argumentos. El primero de ellos es que la

\footnotetext{
${ }^{1}$ Tanto en esta introducción como en algunos de los artículos incluidos en el presente volumen, opto por preservar la etiqueta original en inglés para referirse a tal teoría sobre la naturaleza de los particulares.
}

Revista de Humanidades de Valparaíso, 2020, No 16, 7-16 
existencia de éstos nos proporcionaría los recursos necesarios para desarrollar una teoría de particulares que los identificase con bundles de universales. ${ }^{2}$ El segundo es que aceptar universales indiscernibles nos permitiría responder a las objeciones que usualmente se han presentado a la explicación para la semejanza de los universales cuantitativos que ofrece Armstrong (1978), posiblemente el defensor más prominente de los universales inmanentes en la literatura metafísica contemporánea. Como consecuencia de la posibilidad de universales inmanentes indiscernibles, Rodríguez-Pereyra sostendrá en la última sección de su artículo que debemos abandonar el criterio de distinción entre particulares y universales de acuerdo con el cual la indiscernibilidad de entidades perfectamente semejantes es solamente posible en el caso de los particulares y no el caso de los universales.

Por su parte, el foco principal de Robert García en "La Bundle Theory y el Desafío del Carácter Denso" está en dar explicación al fenómeno, en apariencia evidente, de que un objeto pueda estar caracterizado de muchas maneras. Tal fenómeno se denomina en la literatura contemporánea el fenómeno del carácter denso (thick character) y se considera como uno de los explananda principales de las disputas acerca de la naturaleza de objetos y propiedades. La explicación que García ofrecerá para el fenómeno del carácter denso pasa por defender una versión de la Bundle Theory de propiedades no-compartibles o tropos. En este punto, García discreparía entonces de Rodriguez-Pereyra, pues éste último de acuerdo con García defendería una Bundle Theory Realista. En tal versión de la Bundle Theory, lo que tenemos son bundles de propiedades universales que son compartibles por múltiples objetos particulares, en cuanto es la misma propiedad la que caracteriza a tal multiplicidad de objetos. García sostiene que la versión más robusta de Bundle Theory es la Bundle Theory de tropos $(2014 ; 2014 a)$ y por tanto será desde esa teoría que se buscará despejar la pregunta acerca del carácter denso y ofrecer la respuesta más viable desde tal posición. La pregunta en cuestión, según García, puede formularse explícitamente del siguiente modo y en dos partes: (i) En orden a explicar el carácter denso, ¿cómo debería formularse una bundle theory de tropos? ¿Cuáles son los requerimientos que una bundle theory de tropos debe satisfacer a la hora de explicar adecuadamente el carácter denso?; (ii) Una bundle theory de tropos que satisface tales requerimientos, ¿es preferible a sus rivales? Puesto que para García una respuesta satisfactoria a (i) vuelve prácticamente trivial la respuesta que demos (ii), su esfuerzo argumentativo irá precisamente dirigido a dar cuenta de la primera pregunta.

\section{Consideraciones finales}

En suma, esperamos que el presente número ofrezca al lector una ventana hacia la riqueza y diversidad que es posible encontrar dentro del panorama de la discusión metafísica contemporánea analítica. Si bien la idea que el lector se podrá formar será siempre

\footnotetext{
${ }^{2}$ Más detalles sobre la Bundle Theory pueden encontrarse en el siguiente párrafo.
} 
limitada y parcial, es de esperar que sea posible dar cuenta fidedignamente de la buena salud en la que se encuentran los debates en los que toman parte los autores que contribuyen a este volumen, la relevancia para la metafísica y otras disciplinas de las preguntas que los animan y las posibles perspectivas de desarrollo que dichos debates puedan tener en el futuro.

\section{Referencias bibliográficas}

Adams, R.M. (1974). Theories of Actuality. Noûs, 8, 211-31. https://doi. org/10.2307/2214751

Adams, R.M. (1979). Primitive Thisness and Primitive Identity. Journal of Philosophy, 76, 5-26. https://doi.org/10.2307/2025812

Armstrong, D.M. (1989). A Combinatorial Theory of Possibility. Cambridge: Cambridge University Press. https://doi.org/10.1017/CBO9781139172226

Armstrong, D.M. (1978). A Theory of Universals (Vol. 2 of Universals and Scientific Realism). Cambridge: Cambridge University Press.

Audi, P. (2012). A Clarification and Defense of the Notion of Grounding. En F. Correia y B. Schneider (eds.), Metaphysical Grounding: Understanding the Structure of Reality, pp. 101-121. Cambridge: Cambridge University Press. https://doi.org/10.1017/CBO9781139149136.004

Ayer, A.J. (1936). Language, Truth, and Logic. Londres: Gollancz.

Barcan, R. (1946). A Functional Calculus of First Order Based on Strict Implication. Journal of Symbolic Logic, 11, 1-16. https://doi.org/10.2307/2269159.

Baxter, D. L. M. (1988). Many-one Idetity. Philosophical Papers, 17, 193-216. https://doi.org/10.1080/05568648809506300

Baxter, D. L. M. (2014). Identity, Discernibility, and Composition. En A. J. Cotnoir y D. L. M. Baxter (eds.), Composition as Identity, pp. 244253. Oxford: Oxford University Press. https://doi.org/10.1093/acprof:oso/9780199669615.001.0001

Bennett, K. (2004). Spatio-temporal Coincidence and the Grounding Problem. Philosophical Studies, 118, 339-71. https://doi.org/10.1023/ B:PHIL.0000026471.20355.54

Black, M. (1952). The identity of indiscernibles. Mind, 61, 153-164. https://doi. org/10.1093/mind/LXI.242.153

Bliss, R. y Trogdon, K. (2014). Metaphysical Grounding. Stanford Encyclopaedia for Philosophy. https://plato.stanford.edu/entries/grounding/. Acceso: 05/09/2020. 
Bohn, E. (2014). Unrestricted composition as identity. En A. J. Cotnoir y D. L. M. Baxter (eds.), Composition as Identity, pp. 143-165. Oxford: Oxford University Press. https://doi.org/10.1093/acprof:oso/9780199669615.001.0001

Brewer, B. y Cumpa, J. (2019). The Nature of Ordinary Objects. Cambridge: Cambridge University Press. https://doi.org/10.1017/9781316612897

Bricker, P. (2016). Composition as a Kind of Identity. Inquiry, 59, 264-294. https:// doi.org/10.1080/0020174X.2015.1040447

Campdelacreu, M. (2016). Sutton's Solution to the Grounding Problem and Intrinsically Composed Colocated Objects. Crítica, 48, 77-92. https://doi. org/10.22201/iifs.18704905e.2016.223

Carnap, R. (1928). Der Logische Aufbau der Welt. Leipzig: Felix Meiner Verlag.

Chalmers, D., Manley, D. Wasserman, D. (eds.) (2009). Metametaphysics: New Essays on the Foundations of Ontology. Oxford: Oxford University Press.

Chisholm, Roderick. (1976). Person and Object: A Metaphysical Study. La Salle, Illinois: Open Court Publishing Co.

Correia, F. y Schnieder, B. (eds.) (2012). Metaphysical Grounding: Understanding the Structure of Reality. Cambridge: Cambridge University Press. https://doi. org/10.1017/CBO9781139149136

Correia, F. (2010). Grounding and Truth-Functions. Logique Et Analyse, 53(211), 251-279. https://doi.org/10.2307/44084957

Cotnoir,A.andBaxter, D. (eds.)(2014). Composition as Identity. Oxford:OxfordUniversity Press. https://doi.org/10.1093/acprof:oso/9780199669615.001.0001

Fine, K. (2003). The Non-Identity of a Material Thing and Its Matter. Mind, 112, 195-234. https://doi.org/10.1093/mind/112.446.195

Fine, K. (2008). I-Kit Fine: Coincidence and Form. Aristotelian Society Supplementary Volume, 82, 101-118. https://doi.org/10.1111/j.14678349.2008.00164.X

Fine, K. (2012). A Guide to Ground. EnF. Correia y B. Schneider(eds.), Metaphysical Grounding: Understanding the Structure of Reality, 37-80. Cambridge: Cambridge University Press. https://doi.org/10.1017/CBO9781139149136.002

Frege, G. (1891). Funktion und Begriff. Vortrag, gehalten in der Sitzung vom 9. Januar 1891 der Jenaischen Gesellschaft für Medizin und Naturwissenschaft. Jena: Hermann Pohle.

Frege, G. (1892). Über Begriff und Gegenstand. Vierteljahresschrift für wissenschaftliche Philosophie, 16, 192-205.

Garcia, R. (2014). Bare Particulars and Constituent Ontology. Acta Analytica, 29(2), 149-159. https://doi.org/10.1007/s12136-013-0208-2 
Garcia, R. (2014a). Bundle Theory's Black Box: Gap Challenges for the Bundle Theory of Substance. Philosophia, 42 (1), 115-26. https://doi.org/10.1007/ s11406-013-9466-X

Gilmore, C. (2018). Location and Mereology. Stanford Encyclopaedia for Philosophy. http://plato.stanford.edu/entries/location-mereology/. Acceso: 05/09/20.

Kleinschmidt, S. (ed.) (2014). Mereology and Location. Oxford: Oxford University Press. https://doi.org/10.1093/acprof:oso/9780199593828.001.0001

Korman, D. (2015). Objects. Nothing out of the Ordinary, Oxford: Oxford University Press. https://doi.org/10.1093/acprof:oso/9780198732532.001.0001

Kripke, S. (1959). A Completeness Theorem in Modal Logic. Journal of Symbolic Logic, 24, 1-14. https://doi.org/10.2307/2964568

Kripke, S. (1963). Semantical Considerations on Modal Logic. Acta Philosophica Fennica, 16, 83-94.

Kripke, S. (1971). Identity and Necessity. En M.K. Munitz (ed.), Identity and Individuation, pp. 135-64. New York: New York University Press.

Kripke, S. (1980). Naming and Necessity. Cambridge (MA): Harvard University Press.

Lewis, D. (1973). Counterfactuals. Cambridge (MA): Harvard University Press.

Lewis, D. (1986). On the Plurality of Worlds. Oxford: Blackwell.

Plantinga, A. (1974). The Nature of Necessity. Oxford: Oxford University Press. https://doi.org/10.1093/0198244142.001.0001

Quine, W.V.O. (1953). On What There Is. En From a Logical Point of View, pp. 1-19. Cambridge (MA): Harvard University Press.

Quine, W.V.O. (1953a). Reference and Modality. En From a Logical Point of View, pp. 139-159. Cambridge (MA): Harvard University Press.

Ramsey, F. (1925). Universals. Mind, 34, 401-17. https://doi.org/10.2307/2249716

Russell, B. (1912). The Problems of Philosophy. London: Williams and Norgate.

Russell, B. (1912a). On the Relations of Universals and Particulars. Proceedings of the Aristotelian Society, 12, 1-24. https://doi.org/10.2307/4543817

Rosen, G. (2010). Metaphysical Dependence: Grounding and Reduction. En B. Hale y A. Hoffmann (Eds.), Modality: Metaphysics, Logic, and Epistemology, pp. 109-36. Oxford: Oxford University Press. https://doi.org/10.1093/ acprof:oso/9780199565818.003.0007

Schaffer, J. (2009). On What Grounds What. En D. Chalmers, D. Manley y R. Wasserman (eds.), Metametaphysics: New Essays on the Foundations of Ontology, pp. 347-383. Oxford: Clarendon Press. 
Saenz, N. B. (2015). A Grounding Solution to the Grounding Problem. Philosophical Studies, 172, 2193 - 2214. https://doi.org/10.1007/s11098-014-0405-X

Sider, T. (2001). Four-Dimensionalism: An Ontology of Persistence and Time. Oxford: Clarendon Press. https://doi.org/10.1093/019924443X.001.0001

Sutton, C.S. (2012). Colocated Objects, Tally-Ho: A Solution to the Grounding Problem. Mind, 121, 703-30. https://doi.org/10.1093/mind/fzs078

van Inwagen, P. (1981). The Doctrine of Arbitrary Undetached Parts. Pacific Philosophical Quarterly, 62, 123-137. https://doi.org/10.1111/j.1468-0114.1981. tb00051.x

van Inwagen, P. (1990). Material Beings. Ithaca: Cornell University Press. https:// doi.org/10.7591/9781501713033

Varzi, A. (2020). Mereology. Stanford Encyclopaedia for Philosophy. http://plato. stanford.edu/entries/mereology/. Acceso: 19/03/20.

Williams, D. C. (1953). On the Elements of Being: I. The Review of Metaphysics, 7 (1), 3-18. https://doi.org/10.2307/20123348.

Wallace, M. (2014). Composition as Identity, Modal Parts, and Mereological Essentialism. En A. Cotnoir y D. Baxter (eds.), Composition as Identity, pp. 111-129. Oxford: Oxford University Press. https://doi.org/10.1093/acprof:oso/9780199669615.001.0001

Wallace, M. (2011). “Composition as Identity: I-II”. Philosophy Compass, 6, 804816, 817-827. https://doi.org/doi.org/10.1111/j.1747-9991.2011.00431.x 